\title{
Some Stability Properties of Parametric Quadratically Constrained Nonconvex Quadratic Programs in Hilbert Spaces
}

\author{
V. V. Dong* \\ accepted for publication in AMV, 09/6/2017
}

\begin{abstract}
Stability of nonconvex quadratic programming problems under finitely many convex quadratic constraints in Hilbert spaces is investigated. We present several stability properties of the global solution map, and the continuity of the optimal value function, assuming that the problem data undergoes small perturbations.
\end{abstract}

Mathematics Subject Classification (2010). 90C20, 90C30, 90C31. Key Words. quadratic program in Hilbert spaces, convex quadratic constraints, solution existence, Legendre form, recession cone, solution set, solution map, optimal value function.

\section{Introduction}

Let $\mathcal{H}$ be a real Hilbert space with an inner product $\langle\cdot, \cdot\rangle$ and its induced norm denoted by $\|\cdot\|$. Let $\mathcal{L}(\mathcal{H})$ be the space of continuous linear operators from $\mathcal{H}$ into $\mathcal{H}$ equipped with the operator norm induced by the vector norm in $\mathcal{H}$ and also denoted by $\|\cdot\|$. The norm in the product space $X_{1} \times \ldots \times X_{k}$ of the normed spaces $X_{1}, \ldots, X_{k}$ is defined by $\left\|\left(x_{1}, \ldots, x_{k}\right)\right\|=\max \left\{\left\|x_{1}\right\|, \ldots,\left\|x_{k}\right\|\right\}$. Let

$$
\Omega:=\mathcal{L}(\mathcal{H}) \times \mathcal{H} \times \mathcal{L}(\mathcal{H})^{m} \times \mathcal{H}^{m} \times \mathbb{R}^{m} .
$$

Consider the following quadratic programming problem

$$
\left\{\begin{array}{l}
\min f(x, \omega):=\frac{1}{2}\langle x, T x\rangle+\langle c, x\rangle \\
\text { s. t. } x \in \mathcal{H}: g_{i}(x, \omega):=\frac{1}{2}\left\langle x, T_{i} x\right\rangle+\left\langle c_{i}, x\right\rangle+\alpha_{i} \leqslant 0, i=1, \ldots, m
\end{array}\right.
$$

where $\omega=\left(T, c, T_{1}, \ldots, T_{m}, c_{1}, \ldots, c_{m}, \alpha_{1}, \ldots, \alpha_{m}\right) \in \Omega, T: \mathcal{H} \rightarrow \mathcal{H}$ is a continuous linear selfadjoint operator, $T_{i}$ is a positive semidefinite continuous linear self-adjoint operator on $\mathcal{H}, c, c_{i} \in \mathcal{H}$ and $\alpha, \alpha_{i}$ are real numbers, $i=1,2, \ldots, m$.

\footnotetext{
${ }^{*}$ V. V. Dong, Phuc Yen College of Industry, Vietnam, vuvdong@gmail.com
} 
The constraint set and the solution set of $\left(Q P_{\omega}\right)$ will be denoted by $F(\omega)$ and $\left.\operatorname{Sol} Q P_{\omega}\right)$, respectively. Since $g_{i}, i=1, \ldots, m$, are continuous and convex, $F(\omega)$ is closed and convex. Hence, by Theorem 2.23 in [3, p. 24], the constraint set $F(\omega)$ of $\left(Q P_{\omega}\right)$ is convex and weakly closed.

The recession cone of the constraint set of $\left(Q P_{\omega}\right)$ can be described explicitly as follows (the proof is similar to the proof of Lemma 1.1 in [11]): If $F(\omega)$ is nonempty, then

$$
0^{+} F(\omega)=\left\{v \in \mathcal{H} \mid T_{i} v=0,\left\langle c_{i}, v\right\rangle \leqslant 0, \forall i=1, \ldots, m\right\}
$$

The function

$$
\varphi: \Omega \rightarrow \mathbb{R} \cup\{+\infty\}
$$

defined by

$$
\varphi(\omega)= \begin{cases}\inf \{f(x, \omega): x \in F(\omega)\} & \text { if } F(\omega) \neq \emptyset \\ +\infty & \text { if } F(\omega)=\emptyset\end{cases}
$$

is called the optimal value function of the parametric problem $Q P_{\omega}$.

Quadratic programming problems (QP problems, in short) have been studied fairly completely in the setting of Euclidean spaces; see [12] and the references therein. For infinite dimensional spaces, it was extended to Hilbert spaces. Existence of the solutions for QP problems in Hilbert spaces have been investigated extensively in various versions; see [3, 13-15] and the references therein.

Stability is an important topic in optimization theory and practical applications. The continuity of the solution set mappings and of the optimal value function in parametric optimization problems have been intensively studied in literatures; see, e.g., [2, 3] and the references therein. Bonnans and Shapiro [3] gave sufficient conditions for the upper semicontinuity of the solution set mapping and continuity of the optimal value function in parametric optimization problems by assuming that the level set is nonempty and contained in a compact set. Berge [2] gave a sufficient condition for semicontinuity of the optimal value function in parametric optimization problems.

Since QP problems form a subclass of nonlinear optimization problems, the stability results in nonlinear optimization can be applied to QP problems in Hilbert spaces. However, the special structure of QP problems allows one to have deeper and sharper results on stability properties of QP problems.

This paper studies parametric quadratic programming problems in a Hilbert space. The main results of the paper concern continuity properties of the solution map and the optimal value function of the problem whose quadratic part of the objective function is a Legendre form and the constraints are convex under Slater's condition. Our results can be seen as an extension of those in [12, 16, 17] and the references therein to Hilbert spaces.

The paper is organized as follows. In Section 2 we study continuity of the solution map in a parametric QP problem. Continuity properties of the optimal value function of the problem $\left(Q P_{\omega}\right)$ under a perturbation are investigated in Section 3. 


\section{Continuity of the Global Solution Map}

In this section, we are going to study continuity properties of the solution set mapping $\operatorname{Sol}(\cdot): \Omega \rightrightarrows$ $\mathcal{H}$ of $Q P_{\omega}$ defined by

$$
\operatorname{Sol}(\omega)=\{x \in F(\omega) \mid f(x, \omega)=\varphi(\omega)\}
$$

Definition 2.1. Let $S: X \rightrightarrows Y$ be a set-valued map from Hilbert space $X$ to Hilbert space $Y$. It is said that $S$ is upper semicontinuous (usc) at $\bar{u} \in X$ if for each open set $V \subset Y$ satisfying $S(\bar{u}) \subset V$, there exists $\varepsilon>0$ such that $S(u) \subset V$ whenever $\|u-\bar{u}\|<\varepsilon$. If for each open set $V \subset Y$ satisfying $S(\bar{u}) \cap V \neq \emptyset$ there exists $\varepsilon>0$ such that $S(u) \cap V \neq \emptyset$ whenever $\|u-\bar{u}\|<\varepsilon$, then $S$ is said to be lower semicontinuous (lsc) at $\bar{u} \in X$. If $S$ is simultaneously usc and lsc at $\bar{u}$, we say that it is continuous at $\bar{u}$.

The inequality system $g_{i}(x, \omega) \leqslant 0, i=1, \ldots, m$, is called regular if there exists $x^{0} \in \mathcal{H}$ such that $g_{i}\left(x^{0}, \omega\right)<0, i=1, \ldots, m$.

In this paper, we will only consider the continuous quadratic forms in the form $Q(x)=\langle x, T x\rangle$, where $T: \mathcal{H} \rightarrow \mathcal{H}$ is a continuous linear self-adjoint operator.

Definition 2.2. (see [9, p. 551]) A quadratic form $Q: \mathcal{H} \rightarrow \mathbb{R}$ is said to be a Legendre form if it is weakly lower semicontinuous and $x_{k} \rightarrow x_{0}$ whenever $x_{k}$ weakly converges to $x_{0}$ and $Q\left(x_{k}\right) \rightarrow Q\left(x_{0}\right)$.

It is clear that in the case where $\mathcal{H}$ is of finite dimension, any quadratic form $Q(x)$ on $\mathcal{H}$ is a Legendre form. It is easy to see that on infinite dimensional Hilbert spaces, the quadratic form $\langle x, I x\rangle$ is a Legendre form while the identity operator $I$ is noncompact, the quadratic form $\langle x, 0 x\rangle$ is not a Legendre form while the zero operator 0 is compact.

For each problem $\left.Q P_{\omega}\right)$, we consider the following problem

$$
\min \left\{\frac{1}{2}\langle v, T v\rangle: v \in 0^{+} F(\omega)\right\} .
$$

Let us denote by $\operatorname{Sol} Q P R_{\omega}$ the solution set of $\left.Q P R_{\omega}\right)$. The problem $Q P R_{\omega}$ is closely related to $Q P_{\omega}$. The solution set of $Q P R_{\omega}$ plays an important role in the study of the stability of the problem $Q P_{\omega}$.

The following lemmas will be useful in the sequel.

Lemma 2.1. Suppose that $x^{k} \in F(\omega) \backslash\{0\}$ for all $k,\left\|x^{k}\right\| \rightarrow \infty$ as $k \rightarrow \infty$ and $\left\|x^{k}\right\|^{-1} x^{k}$ weakly converges to $\bar{v}$. Then, $\bar{v} \in 0^{+} F(\omega)$.

Proof. Since $x^{k} \in F(\omega)$, we have

$$
\frac{1}{2}\left\langle x^{k}, T_{i} x^{k}\right\rangle+\left\langle c_{i}, x^{k}\right\rangle+\alpha_{i} \leqslant 0 \quad i=1, \ldots, m
$$


Multiplying both sides of the inequalities in (1) by $\left\|x^{k}\right\|^{-2}$ and letting $k \rightarrow \infty$, we obtain

$$
\liminf _{k \rightarrow \infty} \frac{1}{2}\left\langle\frac{x^{k}}{\left\|x^{k}\right\|}, T_{i} \frac{x^{k}}{\left\|x^{k}\right\|}\right\rangle \leqslant 0, i=1, \ldots, m .
$$

Since $T_{i}$ is positive semidefinite, by Proposition 3 in [10, p. 269], $\left\langle x, T_{i} x\right\rangle$ is weakly lower semicontinuous. Hence,

$$
\frac{1}{2}\left\langle\bar{v}, T_{i} \bar{v}\right\rangle \leqslant \liminf _{k \rightarrow \infty} \frac{1}{2}\left\langle\frac{x^{k}}{\left\|x^{k}\right\|}, T_{i} \frac{x^{k}}{\left\|x^{k}\right\|}\right\rangle \leqslant 0 \quad i=1, \ldots, m .
$$

By the positive semideniteness of $T_{i}$, from this we can deduce that

$$
T_{i} \bar{v}=0 \quad \forall i=1, \ldots, m
$$

As $\left\langle x^{k}, T_{i} x^{k}\right\rangle \geqslant 0$, from (11) it follows that

$$
\left\langle c_{i}, x^{k}\right\rangle+\alpha_{i} \leqslant 0, \quad \forall i=1, \ldots, m, \quad \forall k .
$$

Multiplying the inequality $\left\langle c_{i}, x^{k}\right\rangle+\alpha_{i} \leqslant 0$ by $\left\|x^{k}\right\|^{-1}$ and letting $k \rightarrow \infty$, we get

$$
\left\langle c_{i}, \bar{v}\right\rangle \leqslant 0 \quad \forall i=1, \ldots, m
$$

Combining (2) with (3) we obtain $\bar{v} \in 0^{+} F(\omega)$.

Lemma 2.2. Consider the problem $Q P_{\omega}$, where $\langle x, T x\rangle$ is a Legendre form. Suppose that $F(\omega)$ is nonempty and $\operatorname{Sol} Q P R_{\omega}=\{0\}$. Then, $\operatorname{Sol}(\omega)$ is a nonempty, closed and bounded set.

Proof. We first prove that $\operatorname{Sol}(\omega)$ is a nonempty set. By [8, Theorem 2], it suffices to show that $f(x, \omega)$ is bounded from below over $F(\omega)$. On the contrary, suppose that $f(x, \omega)$ is unbounded from below over $F(\omega)$. Then, there exists a sequence $\left\{x^{k}\right\} \subset F(\omega)$ such that $f\left(x^{k}\right) \rightarrow-\infty$ as $k \rightarrow \infty$. If $\left\{x^{k}\right\}$ is bounded, it has a weakly convergent subsequence. Without loss of generality, we can assume that $x^{k}$ itself weakly converges to some $\bar{x}$. By the weakly closedness of $F(\omega)$, we have $\bar{x} \in F(\omega)$. Since $\langle x, T x\rangle$ is a Legendre form, it is weakly lower semicontinuous, one has $f(\bar{x}) \leqslant \liminf _{k \rightarrow \infty} f\left(x^{k}\right)=-\infty$, which is impossible. Hence $\left\{x^{k}\right\}$ is unbounded. Since $f\left(x^{k}\right) \rightarrow-\infty$ as $k \rightarrow \infty$ and $\left\{x^{k}\right\}$ is unbounded, by taking a subsequence, if necessary, we may assume that

$$
f\left(x^{k}, \omega\right)=\frac{1}{2}\left\langle x^{k}, T x^{k}\right\rangle+\left\langle c, x^{k}\right\rangle \leqslant 0
$$

for all $k,\left\|x^{k}\right\| \neq 0,\left\|x^{k}\right\| \rightarrow \infty$ as $k \rightarrow \infty$, and $v^{k}:=\left\|x^{k}\right\|^{-1} x^{k}$ weakly converges to some $\bar{v}$ as $k \rightarrow \infty$. It follows from Lemma 2.1 that $\bar{v} \in 0^{+} F(\omega)$. Multiplying both sides of (4) by $\left\|x^{k}\right\|^{-2}$ and letting $k \rightarrow \infty$, one has

$$
\langle\bar{v}, T \bar{v}\rangle \leqslant \liminf _{k \rightarrow \infty} \frac{1}{2}\left\langle v^{k}, T v^{k}\right\rangle \leqslant \limsup _{k \rightarrow \infty} \frac{1}{2}\left\langle v^{k}, T v^{k}\right\rangle \leqslant 0
$$


We next claim that $\bar{v} \neq 0$. Indeed, if $\bar{v}=0$, then it follows from (5) that $\left\langle v^{k}, T v^{k}\right\rangle \rightarrow\langle\bar{v}, T \bar{v}\rangle$ as $k \rightarrow \infty$. Since $\langle x, T x\rangle$ is a Legendre form, and since $v^{k} \rightarrow \bar{v},\left\langle v^{k}, T v^{k}\right\rangle \rightarrow\langle\bar{v}, T \bar{v}\rangle$ as $k \rightarrow \infty$, by Definition 2.2 we deduce that $v^{k}$ converges to $\bar{v}$ and $\bar{v} \neq 0$. Consequently, we have shown that there exists $\bar{v} \neq 0$ such that $\bar{v} \in 0^{+} F(\omega)$ and $\langle\bar{v}, T \bar{v}\rangle \leqslant 0$. But this contradicts our assumption that $\left.\operatorname{Sol} Q P R_{\omega}\right)=\{0\}$. Hence $f(x, \omega)$ is bounded from below over $F(\omega)$ and we have $\operatorname{Sol}(\omega)$ is a nonempty set.

The closedness of $\operatorname{Sol}(\omega)$ is evident because $f(x, \omega)$ is weakly lower semicontinuous and $F(\omega)$ is a closed convex set.

We next prove that $\operatorname{Sol}(\omega)$ is bounded. Suppose that $\operatorname{Sol}(\omega)$ is unbounded. Then, there exists $\left\{y^{k}\right\} \subset \operatorname{Sol}(\omega)$ such that $\left\|y^{k}\right\| \rightarrow \infty$ as $k \rightarrow \infty$. Without loss generality we may suppose that $\left\|y^{k}\right\| \neq 0$. Let $v^{k}:=\frac{y^{k}}{\left\|y^{k}\right\|}$, one has $\left\|v^{k}\right\|=1$. Since $\mathcal{H}$ is Hilbert space, extracting if necessary a subsequence, we may assume that $v^{k}$ itself weakly converges to some $\bar{v}$. It follows from Lemma 2.1 that $\bar{v} \in 0^{+} F(\omega)$.

Fixing any $x \in F(\omega)$, one has

$$
\frac{1}{2}\left\langle y^{k}, T y^{k}\right\rangle+\left\langle c, y^{k}\right\rangle \leqslant \frac{1}{2}\langle x, T x\rangle+\langle c, x\rangle
$$

Since $\langle x, T x\rangle$ is a Legendre form, it is weakly lower semicontinuous. Dividing both sides of (66) by $\left\|y^{k}\right\|^{2}$ and letting $k \rightarrow \infty$, we get

$$
\langle\bar{v}, T \bar{v}\rangle \leqslant \liminf _{k \rightarrow \infty}\left\langle v^{k}, T v^{k}\right\rangle \leqslant \limsup _{k \rightarrow \infty}\left\langle v^{k}, T v^{k}\right\rangle \leqslant 0
$$

By a similar argument to the one given above, we have $\bar{v} \in 0^{+} F(\omega) \backslash\{0\}$ and $\langle\bar{v}, T \bar{v}\rangle \leqslant 0$, contrary to $\left.\operatorname{Sol} Q P R_{\omega}\right)=\{0\}$. Hence $\operatorname{Sol}(\omega)$ is bounded.

Note that, the problem $Q P_{\omega}$ may have no solution if the assumption on the Legendre property of the quadratic form is omitted (see [7, Example 3.3]).

The next example shows that the conclusion of Lemma 2.2 fails if the assumption on the Legendre property of the quadratic form is omitted.

Example 2.1. Consider the programming problem

$$
\left\{\begin{array}{l}
\min f(x, \omega)=\frac{1}{2}\langle x, T x\rangle \\
\text { s. t. } x \in L_{2}[0,1], g_{1}(x, \omega)=\frac{1}{2}\left\langle x, T_{1} x\right\rangle+\alpha_{1} \leqslant 0
\end{array}\right.
$$

where $T=0, T_{1}: L_{2}[0,1] \rightarrow L_{2}[0,1]$ is defined by $T_{1} x=t x(t), \alpha_{1}=-\frac{1}{4}$ and $\omega=\left(T, T_{1}, \alpha_{1}\right)$. It is easily seen that $\langle x, T x\rangle=\langle x, 0 x\rangle$ is not a Legendre form.

Let $F(\omega)=\left\{x \in L_{2}[0,1] \mid g_{1}(x, \omega)=\frac{1}{2} \int_{0}^{1} t x^{2}(t) d t-\frac{1}{4} \leqslant 0\right\}$.

It is easy to check that $F(\omega) \neq \emptyset$ and

$$
0^{+} F(\omega)=\left\{v \in L_{2}[0,1] \mid t v(t)=0 \forall t \in[0,1]\right\}=\{0\}
$$


Therefore $\operatorname{Sol}\left(Q P R_{\omega}\right)=\{0\}$.

Let

$$
x_{k}(t)=\left\{\begin{array}{lll}
k & \text { if } & 0 \leqslant t \leqslant \frac{1}{k} \\
0 & \text { if } & \frac{1}{k}<t \leqslant 1
\end{array}\right.
$$

it is easy to check that $x_{k}(t) \in L_{2}[0,1]$.

We have $g_{1}\left(x_{k}, \omega\right)=\frac{1}{2} \int_{0}^{\frac{1}{k}} t k^{2} d t-\frac{1}{4}=0$ and $\left\|x_{k}\right\|^{2}=\int_{0}^{\frac{1}{k}} k^{2} d t=k$. Hence $x_{k} \in F(\omega)$ and $\left\|x_{k}\right\| \rightarrow \infty$ as $k \rightarrow \infty$.

Since $f(x, \omega)=0$ for all $x \in F(\omega)$, it follows that the solution set of (8) coincides with $F(\omega)$. Thus the solution set of (8) is unbounded.

Corollary 2.1. Consider the problem $\left(Q P_{\omega}\right)$, where $\langle x, T x\rangle$ is a nonnegative Legendre form. Assume that $F(\omega)$ is nonempty and $\operatorname{Sol}\left(Q P R_{\omega}\right)=\{0\}$. Then, $\operatorname{Sol}(\omega)$ is nonempty and weakly compact.

Proof. It follows from Lemma 2.2 that $\operatorname{Sol}(\omega)$ is a nonempty and bounded set. Since $\langle x, T x\rangle$ is nonnegative, it follows that $\left.Q Q_{\omega}\right)$ is a convex problem. Hence $\operatorname{Sol}(\omega)$ is a convex set. By Theorem 3.3 in [1], $\operatorname{Sol}(\omega)$ is weakly compact.

Lemma 2.3. [6, Theorem 5.1] Let $\omega \in \Omega$. If the system $g_{i}(x, \omega) \leqslant 0, i=1$, dots, $m$, is regular, then the set-value map $F(\cdot): \Omega \rightrightarrows \mathcal{H}$ is defined by $F\left(\omega^{\prime}\right)=\left\{x \in \mathcal{H} \mid g_{i}\left(x, \omega^{\prime}\right) \leqslant 0, i=1, \ldots, m\right\}$ is lower semicontinuous at $\omega$.

Remark 2.1. If the inequality system $g_{i}(x, \omega) \leqslant 0, i=1, \ldots, m$, is irregular, then there exists a sequence $\left\{\omega^{k}\right\}$ in $\Omega$ converging to $\omega$ such that, for every $k$, the system $g_{i}\left(x, \omega^{k}\right) \leqslant 0, i=1, \ldots, m$, has no solution.

Lemma 2.4. Consider the parametric quadratic function on Hilbert space $\mathcal{H}$

$$
h(x, u):=\frac{1}{2}\langle x, T x\rangle+\langle c, x\rangle+\alpha,
$$

where $\langle x, T x\rangle$ is weakly lower semicontinuous, $c \in \mathcal{H}, \alpha \in \mathbb{R}$ and $u=(T, c, \alpha) \in \Omega_{1}:=\mathcal{L}(\mathcal{H}) \times \mathcal{H} \times \mathbb{R}$.

Suppose that there exists a sequence $\left\{u^{k}\right\}=\left\{\left(T^{k}, c^{k}, \alpha^{k}\right)\right\}$ in $\Omega_{1}$ converging to $u$ and $x^{k}$ weakly converges to $\bar{x}$ as $k \rightarrow \infty$. Then,

(a) $\liminf _{k \rightarrow \infty} h\left(x^{k}, u^{k}\right) \geqslant h(\bar{x}, u)$;

(b) If $\left\langle x^{k}, T^{k} x^{k}\right\rangle \rightarrow\langle\bar{x}, T \bar{x}\rangle$ as $k \rightarrow \infty$ then $\left\langle x^{k}, T x^{k}\right\rangle \rightarrow\langle\bar{x}, T \bar{x}\rangle$ as $k \rightarrow \infty$;

(c) If $T^{k} x^{k}=0,\left\langle c^{k}, x^{k}\right\rangle \leqslant 0$ and $\langle x, T x\rangle$ is nonnegative, then

$$
T \bar{x}=0,\langle\bar{c}, \bar{x}\rangle \leqslant 0 .
$$


Proof. (a) Let us first prove that $\liminf _{k \rightarrow \infty}\left\langle x^{k}, T^{k} x^{k}\right\rangle \geqslant\langle\bar{x}, T \bar{x}\rangle$. We have

$$
\left\langle x^{k}, T^{k} x^{k}\right\rangle-\langle\bar{x}, T \bar{x}\rangle=\left\langle x^{k},\left(T^{k}-T\right) x^{k}\right\rangle+\left[\left\langle x^{k}, T x^{k}\right\rangle-\langle\bar{x}, T \bar{x}\rangle\right] .
$$

Since $\left\|T^{k}-T\right\| \rightarrow 0$ as $k \rightarrow \infty$ and since $\left\|x^{k}\right\|$ is bounded, by the Cauchy-Schwarz inequality (see [1, p. 29]) we see that

$$
\left|\left\langle x^{k},\left(T^{k}-T\right) x^{k}\right\rangle\right| \leqslant\left\|x^{k}\right\|^{2}\left\|T^{k}-T\right\| \rightarrow 0 \text { as } k \rightarrow \infty .
$$

By (9), (10) and the weakly lower semicontinuity of $\langle x, T x\rangle$, one has

$$
\liminf _{k \rightarrow \infty}\left\langle x^{k}, T^{k} x^{k}\right\rangle \geqslant\langle\bar{x}, T \bar{x}\rangle .
$$

Combining (11) with $\lim _{k \rightarrow \infty}\left(\left\langle c^{k}, x\right\rangle+\alpha^{k}\right)=\langle c, x\rangle+\alpha$ as $k \rightarrow \infty$ we can assert that $\liminf _{k \rightarrow \infty} h\left(u^{k}, x^{k}\right) \geqslant$ $h(u, \bar{x})$.

(b) Since $\left\langle x^{k}, T x^{k}\right\rangle-\langle\bar{x}, T \bar{x}\rangle=\left\langle x^{k},\left(T-T^{k}\right) x^{k}\right\rangle+\left[\left\langle x^{k}, T^{k} x^{k}\right\rangle-\langle\bar{x}, T \bar{x}]\right.$, it follows that

$$
\left\|\left\langle x^{k}, T x^{k}\right\rangle-\langle\bar{x}, T \bar{x}\rangle\right\| \leqslant\left\|\left\langle x^{k},\left(T-T^{k}\right) x^{k}\right\rangle\right\|+\left\|\left\langle x^{k}, T^{k} x^{k}\right\rangle-\langle\bar{x}, T \bar{x})\right\| .
$$

From (10), (12) and the assumption $\left\langle x^{k}, T^{k} x^{k}\right\rangle \rightarrow\langle\bar{x}, T \bar{x}\rangle$ as $k \rightarrow \infty$, it may be concluded that $\left\langle x^{k}, T x^{k}\right\rangle \rightarrow\langle x, T x\rangle$ as $k \rightarrow \infty$.

(c) Obviously, $\langle c, \bar{x}\rangle=\lim _{k \rightarrow \infty}\left\langle c^{k}, x^{k}\right\rangle \leqslant 0$. By the assumption $T^{k} x^{k}=0$ and $\langle x, T x\rangle$ is nonnegative, it follows from (11), that $\langle\bar{x}, T \bar{x}\rangle=0$. By the well-known Fermat Rule, we obtain that $T \bar{x}=0$. The proof is complete.

Lemma 2.5. Consider the problem $\left(Q P_{\omega}\right)$, where $\langle x, T x\rangle$ is a Legendre form. Then, set

$$
K:=\left\{\left(T, T_{1}, \ldots, T_{m}, c_{1}, \ldots, c_{m}\right) \in \mathcal{L}(\mathcal{H})^{m+1} \times \mathcal{H}^{m} \mid \operatorname{Sol}\left(Q P R_{\omega}\right)=\{0\}\right\}
$$

is open in $\mathcal{L}(\mathcal{H})^{m+1} \times \mathcal{H}^{m}$.

Proof. On the contrary, suppose that $K$ is not open. Then, there exists a sequence

$$
\left\{\left(T^{k}, T_{1}^{k}, \ldots, T_{m}^{k}, c_{1}^{k}, \ldots, c_{m}^{k}\right)\right\} \subset \mathcal{L}(\mathcal{H})^{m+1} \times \mathcal{H}^{m}
$$

converging to $\left(T, T_{1}, \ldots, T_{m}, c_{1}, \ldots, c_{m}\right) \in K$ and a sequence $v^{k} \in \mathcal{H},\left\|v^{k}\right\| \neq 0$ such that

$$
T_{i}^{k} v^{k}=0,\left\langle c_{i}^{k}, v^{k}\right\rangle \leqslant 0, \quad i=1, \ldots, m,\left\langle v^{k}, T^{k} v^{k}\right\rangle \leqslant 0 .
$$

Let $h^{k}:=\frac{v^{k}}{\left\|v^{k}\right\|}$. By taking a subsequence, if necessary, we can assume that $h^{k}$ weakly converges to $\bar{v}$. It follows from Lemma 2.4 that $\bar{v} \in 0^{+} F(\omega)$.

Dividing both sides of the inequalities $\left\langle v^{k}, T^{k} v^{k}\right\rangle \leqslant 0$ in (13) by $\left\|v^{k}\right\|^{2}$ and letting $k \rightarrow \infty$, one has

$$
\limsup _{k \rightarrow \infty}\left\langle v^{k}, T^{k} v^{k}\right\rangle \leqslant 0 .
$$


Combining this with Lemma 2.4 we deduce that

$$
\langle\bar{v}, T \bar{v}\rangle \leqslant \liminf _{k \rightarrow \infty}\left\langle v^{k}, T^{k} v^{k}\right\rangle \leqslant \limsup _{k \rightarrow \infty}\left\langle v^{k}, T^{k} v^{k}\right\rangle=0
$$

We next claim that $\bar{v} \neq 0$. Indeed, if $\bar{v}=0$, then it follows from (14) and Lemma 2.4 that $\left\langle v^{k}, T v^{k}\right\rangle \rightarrow\langle\bar{v}, T \bar{v}\rangle$ as $k \rightarrow \infty$. Since $\langle x, T x\rangle$ is a Legendre form, and since $v^{k} \rightarrow \bar{v},\left\langle v^{k}, T v^{k}\right\rangle \rightarrow$ $\langle\bar{v}, T \bar{v}\rangle$ as $k \rightarrow \infty$, by Definition 2.2 we deduce that $v^{k}$ converges to $\bar{v}$ and $\bar{v} \neq 0$, a contradiction.

We have thus proved that there exists $\bar{v} \in 0^{+} F(\omega) \backslash\{0\}$ such that $\langle\bar{v}, T \bar{v}\rangle \leqslant 0$. This contradicts the assumption that $\left.\operatorname{Sol} Q P R_{\omega}\right)=\{0\}$. Hence $K$ is open. The proof is complete.

The next example shows that the assumption on the Legendre property of the quadratic form cannot be dropped from the assumption of Lemma 2.5.

Example 2.2. Consider the programming problem

$$
\left\{\begin{array}{l}
\min f(x, \omega)=\frac{1}{2}\langle x, T x\rangle+\langle c, x\rangle \\
\text { s. t. } x=\left(x_{1}, x_{2}, \ldots\right) \in \ell^{2}, g_{1}(x, \omega)=\frac{1}{2}\left\langle x, T_{1} x\right\rangle+\left\langle c_{1}, x\right\rangle+\alpha_{1} \leqslant 0,
\end{array}\right.
$$

where $T=0, c=0, T_{1}: \ell^{2} \rightarrow \ell^{2}$ is defined by $T_{1} x=\left(x_{1}, \frac{x_{2}}{2^{2}}, \ldots, \frac{x_{n}}{n^{n}}, \ldots\right), c_{1}=0, \alpha_{1}=-1$ and $\omega=\left(T, c, T_{1}, c_{1}, \alpha_{1}\right)$.

Let

$$
F(\omega)=\left\{x \in \ell^{2} \mid g_{1}(x, \omega)=\frac{1}{2}\left\langle x, T_{1} x\right\rangle+\left\langle c_{1}, x\right\rangle+\alpha_{1} \leqslant 0\right\}
$$

and let

$$
\left.K=\left\{\left(T, T_{1}, c_{1}\right) \in \mathcal{L}\left(\ell^{2}\right)^{2} \times \ell^{2} \mid \operatorname{Sol}\left(\underline{Q P R_{\omega}}\right)\right)=\{0\}\right\} .
$$

It is easily seen that $F(\omega)$ is a nonempty set and $\langle x, T x\rangle=\langle x, 0 x\rangle$ is not a Legendre form.

The quadratic form associated with $T_{1}$ given by $\left\langle x, T_{1} x\right\rangle=\sum_{n=1}^{\infty} \frac{x_{n}^{2}}{n^{n}}$. Since $\left\langle x, T_{1} x\right\rangle=\sum_{n=1}^{\infty} \frac{x_{n}^{2}}{n^{n}}=0$ if and only if $x=(0, \ldots, 0, \ldots)$, it follows that

$$
0^{+} F(\omega)=\left\{v \in \ell^{2} \mid T_{1} v=0\right\}=\{0\}
$$

Hence Sol $\left.Q P R_{\omega}\right)=\{0\}$.

Let $T_{1}^{n}=T_{1}-\frac{1}{n^{n}} I$, where $I$ is the identity operator on $\ell^{2}$.

Since $\left\|T_{1}-T_{1}^{n}\right\|=\frac{1}{n^{n}}$, we have $\left\|\left(T, T_{1}^{n}\right)-\left(T, T_{1}\right)\right\|=\left\|T_{1}^{n}-T_{1}\right\| \rightarrow 0$ as $n \rightarrow \infty$. It is clear that $T^{n} \in \ell^{2}$ and

$$
T_{1}^{n} x=\left(x_{1}+\ldots\left(\frac{1}{n^{n-1}}-\frac{1}{n^{n}}\right) x_{n-1}+0+\left(\frac{1}{n^{n+1}}-\frac{1}{n^{n}}\right) x_{n+1}+\ldots\right) \text { for all } n .
$$

Note that if $\bar{v}^{n}=\left(0,0, \ldots, v_{n}, 0, \ldots\right), v_{n} \neq 0$, then $T_{1}^{n} \bar{v}^{n}=0$. Therefore $\operatorname{Sol}\left(Q P R_{\omega^{n}}\right) \neq\{0\}$.

We have shown that there exists a sequence $\left\{\left(T, T_{1}^{n}, c_{1}\right)\right\} \subset \mathcal{L}\left(\ell^{2}\right)^{2} \times \ell^{2}$ converging to $\left(T, T_{1}, c_{1}\right) \in K$ such that $\operatorname{Sol}\left(Q P R_{\omega^{n}}\right) \neq\{0\}$. Hence $K$ is not open. 
A sufficient condition for the upper semicontinuity of $\mathrm{Sol}(\cdot)$ is as follows.

Theorem 2.1. Consider the problem $Q P_{\omega}$ where $\langle x, T x\rangle$ is a Legendre form. Then, the multifunction $\operatorname{Sol}(\cdot)$ is usc at $\omega$ if the following two conditions are satisfied:

(i) $\operatorname{Sol} Q P R_{\omega}=\{0\}$;

(ii) The system $g_{i}(x, \omega) \leqslant 0, i=1, \ldots, m$, is regular.

Proof. Suppose that the assertion of the theorem is false. Then, there exist an open set $V$ containing $\operatorname{Sol}(\omega)$, a sequence $\left\{\omega^{k}\right\}$ converging to $\omega$, a sequence $\left\{x^{k}\right\}$ such that $x^{k} \in \operatorname{Sol}\left(\omega^{k}\right) \backslash V$ for all $k$. Since $x^{k} \in \operatorname{Sol}\left(\omega^{k}\right)$, one has

$$
g_{i}\left(x^{k}, \omega^{k}\right) \leqslant 0, i=1, \ldots, m
$$

Fix any $\bar{x} \in F(\omega)$. By assumption (ii) and Lemma 2.3, there exists a sequence $\left\{\xi^{k}\right\}, \quad \xi^{k} \in F\left(\omega^{k}\right)$ for all $k$ such that $\lim _{k \rightarrow \infty} \xi^{k}=\bar{x}$. We have

$$
\frac{1}{2}\left\langle x^{k}, T^{k} x^{k}\right\rangle+\left\langle c^{k}, x^{k}\right\rangle \leqslant \frac{1}{2}\left\langle\xi^{k}, T^{k} \xi^{k}\right\rangle+\left\langle c^{k}, \xi^{k}\right\rangle
$$

If the sequence $\left\{x^{k}\right\}$ is bounded, then there is no loss of generality in assuming that $x^{k} \rightarrow x_{0} \in \mathcal{H}$. By passing to the limit in (16) and (17) as $k \rightarrow \infty$, and using Lemma 2.4, we obtain $g_{i}\left(x_{0}, \omega\right) \leqslant 0$ and $f\left(x_{0}, \omega\right) \leqslant f(\bar{x}, \omega)$. Hence $x_{0} \in \operatorname{Sol}(\omega) \subset V$. We have arrived at a contradiction, because $x_{0} \notin V$ for all $k$, and $V$ is open.

Suppose now that $\left\{x^{k}\right\}$ is unbounded. Without loss of generality we can assume that $\left\|x^{k}\right\| \neq 0$ for all $k$ and $\left\|x^{k}\right\| \rightarrow \infty$ as $k \rightarrow \infty$. Let $v^{k}:=\frac{x^{k}}{\| x^{k}} \|$, we have $\left\|v^{k}\right\|=1$, by taking a subsequence, if necessary, we can assume that $v^{k} \rightarrow v$ as $k \rightarrow \infty$. It follows from Lemma 2.1 that $v \in 0^{+} F(\omega)$. Dividing both sides of (17) by $\left\|x^{k}\right\|^{2}$ and letting $k \rightarrow \infty$, by Lemma 2.4(a) we can deduce that

$$
\langle v, T v\rangle \leqslant \liminf _{k \rightarrow \infty}\left\langle v^{k}, T^{k} v^{k}\right\rangle \leqslant \limsup _{k \rightarrow \infty}\left\langle v^{k}, T^{k} v^{k}\right\rangle \leqslant 0
$$

In the same way as in the proof of Lemma 2.5, we obtain $v \in 0^{+} F(\omega) \backslash\{0\}$ and $\langle v, T v\rangle \leqslant 0$, a contradiction. The proof is complete.

Remark 2.2. Observe that neither a) nor b) is a necessary condition for the upper semicontinuity of the $\operatorname{Sol}($.$) at a given \omega$ (see, [12, Example 12.1 and 12.2]).

Corollary 2.2. Consider the problem $Q P_{\omega}$, where $\langle x, T x\rangle$ is a Legendre form. Suppose that the system $g_{i}(x, \omega) \leqslant 0, i=1, \ldots, m$, is regular and $F(\omega)$ is bounded. Then, $\operatorname{Sol}(\cdot)$ is usc at $\omega$.

Proof. Since the system $g_{i}(x, \omega) \leqslant 0, i=1, \ldots, m$, is regular, $F(\omega) \neq \emptyset$. The boundedness of $F(\omega)$ implies that $0^{+} F(\omega)=\{0\}$. Hence $\left.\operatorname{Sol} Q P R_{\omega}\right)=\{0\}$, and the desired property follows from Theorem 2.1. The proof is complete. 
The following example shows that the assumption on the Legendre property of the quadratic form cannot be dropped from the assumption of Theorem 2.1.

Example 2.3. Consider the problem

$$
\left\{\begin{array}{l}
\min f(x, \omega)=\frac{1}{2}\langle x, T x\rangle \\
\text { s. t. } x=\left(x_{1}, x_{2}, \ldots\right) \in \ell^{2}, g_{1}(x, \omega)=\left\langle c_{1}, x\right\rangle+\alpha_{1} \leqslant 0
\end{array}\right.
$$

where $T: \ell^{2} \rightarrow \ell^{2}$ is defined by

$$
T x=\left(x_{1}, \frac{x_{2}}{2^{2}}, \ldots, \frac{x_{n}}{n^{n}}, \ldots\right),
$$

$c_{1}=\left(-1,-\frac{1}{2}, \ldots,-\frac{1}{n}, \ldots\right), \alpha_{1}=1$ and $\omega=\left(T, c_{1}, \alpha_{1}\right)$.

Let $F(\omega)=\left\{x \in \ell^{2} \mid g_{1}(x, \omega)=\left\langle c_{1}, x\right\rangle+\alpha_{1} \leqslant 0\right\}$ and let $\operatorname{Sol}(\omega)$ denote the solution of (18).

Since $x=(1,0, \ldots) \in F(\omega)$, we have $F(\omega)$ is a nonempty set.

The quadratic form $\langle x, T x\rangle=\sum_{n=1}^{\infty} \frac{x_{n}^{2}}{n^{n}}$ is not a Legendre form and $\operatorname{Sol}(\omega)=\emptyset$ (see, [7, Example 3.3]). Let $\omega^{\varepsilon}=\left(T^{\varepsilon}, c_{1}, \alpha_{1}\right)$, where $T^{\varepsilon}=T+\varepsilon I, \varepsilon>0$ and $I$ is the identity operator on $\ell^{2}$. Since $\left\|T^{\varepsilon}-T\right\|=\varepsilon$, we have

$$
\left\|\omega^{\varepsilon}-\omega\right\|=\max \left\{\left\|T^{\varepsilon}-T\right\|,\left\|c_{1}-c_{1}\right\|,\left\|\alpha_{1}-\alpha_{1}\right\|\right\}=\left\|T^{\varepsilon}-T\right\| \rightarrow 0 \text { as } \varepsilon \rightarrow 0 .
$$

We have also

$$
\left\langle x, T^{\varepsilon} x\right\rangle=\langle x, T x\rangle+\varepsilon\langle x, I x\rangle=\langle x, T x\rangle+\varepsilon\|x\|^{2} \geqslant \varepsilon\|x\|^{2},
$$

because $\langle x, T x\rangle \geqslant 0$. Hence $\left\langle x, T^{\varepsilon} x\right\rangle$ is a Legendre form.

Consider the problem

$$
\left\{\begin{array}{l}
\min f\left(x, \omega^{\varepsilon}\right)=\frac{1}{2}\left\langle x, T^{\varepsilon} x\right\rangle \\
\text { s. t. } x=\left(x_{1}, x_{2}, \ldots\right) \in \ell^{2}, g_{1}\left(x, \omega^{\varepsilon}\right)=\left\langle c_{1}, x\right\rangle+\alpha_{1} \leqslant 0 .
\end{array}\right.
$$

Let $\operatorname{Sol}\left(\omega^{\varepsilon}\right)$ denote the solution of (19). Since $\left\langle x, T^{\varepsilon} x\right\rangle$ is nonnegative and $\left\langle x, T^{\varepsilon} x\right\rangle=0$ if and only if $x=0$, we have $\operatorname{Sol}\left(Q P R_{\omega^{\varepsilon}}\right)=\{0\}$. It follows from Lemma 2.2 that $\operatorname{Sol}\left(\omega^{\varepsilon}\right)$ is a nonempty set for every $\varepsilon$.

We have shown that there exists a sequence $\left\{\omega^{\varepsilon}\right\}$ converging to $\omega$ such that $\operatorname{Sol}\left(\omega^{\varepsilon}\right) \neq \emptyset$. Taking $V=\emptyset$ we get $\operatorname{Sol}(\omega) \subset V$ and $\operatorname{Sol}\left(\omega^{\varepsilon}\right) \not \subset V$. Hence $\operatorname{Sol}(\cdot)$ is not usc at $\omega=\left(T, c_{1}, \alpha_{1}\right)$.

Lemma 2.6. Suppose that $\langle x, T x\rangle$ is a Legendre form on $\mathcal{H}$. Then, there exists an open neighborhood $\mathcal{U}$ of $T$ in space of continuous linear operators $\mathcal{L}(\mathcal{H})$ such that for every $T^{\prime} \in \mathcal{U},\left\langle x, T^{\prime} x\right\rangle$ is also a Legendre form.

Proof. Since $\langle x, T x\rangle$ is a Legendre form, there exist an elliptic form $\left\langle x, T_{1} x\right\rangle$ and a quadratic form of finite rank $\left\langle x, T_{2} x\right\rangle$ such that $\langle x, T x\rangle=\left\langle x, T_{1} x\right\rangle+\left\langle x, T_{2} x\right\rangle$ (see [3, Proposition 3.79]).

Let $\alpha$ be a positive number such that $\left\langle x, T_{1} x\right\rangle \geqslant \alpha\|x\|^{2}, \forall x \in \mathcal{H}$. Choose $\varepsilon>0$ so that $\varepsilon<\alpha$. Define $\mathcal{U}:=\left\{T^{\prime} \in \mathcal{L}(\mathcal{H}) \mid\left\|T-T^{\prime}\right\|<\varepsilon\right\}$. 
Let $T^{\prime} \in \mathcal{U}$. By the Cauchy-Schwarz inequality (see [1, p. 29]), we obtain

$$
\langle x, T x\rangle-\left\langle x, T^{\prime} x\right\rangle=\left\langle x,\left(T-T^{\prime}\right) x\right\rangle \leqslant\left\|T-T^{\prime}\right\|\|x\|^{2} \leqslant \varepsilon\|x\|^{2} .
$$

Substituting $\langle x, T x\rangle=\left\langle x, T_{1} x\right\rangle+\left\langle x, T_{2} x\right\rangle$ into (20) we obtain

$$
\left\langle x, T^{\prime} x\right\rangle-\left\langle x, T_{2} x\right\rangle \geqslant\left\langle x, T_{1} x\right\rangle-\varepsilon\|x\|^{2} .
$$

Combining (21) with $\left\langle x, T_{1} x\right\rangle \geqslant \alpha\|x\|^{2}$ yields

$$
\left\langle x, T^{\prime} x\right\rangle-\left\langle x, T_{2} x\right\rangle \geqslant(\alpha-\varepsilon)\|x\|^{2} .
$$

From this it follows that $\widetilde{Q}(x):=\left\langle x, T^{\prime} x\right\rangle-\left\langle x, T_{2} x\right\rangle$ is elliptic.

We have shown that the quadratic form $\left\langle x, T^{\prime} x\right\rangle=\widetilde{Q}(x)+\left\langle x, T_{2} x\right\rangle$ is the sum of an elliptic quadratic form and a quadratic form of finite rank. By [3, Proposition 3.79], $\left\langle x, T^{\prime} x\right\rangle$ is a Legendre form. The proof is complete.

The next theorem gives the necessary and sufficient condition for the lower semicontinuity of $\operatorname{Sol}(\cdot)$.

Theorem 2.2. Consider the problem QP $Q P_{\omega}$ where $\langle x, T x\rangle$ is a Legendre form. Then, Sol $(\cdot)$ is lsc at $\omega$ if and only if the following three conditions are satisfied:

(i) $\operatorname{Sol} Q P R_{\omega}=\{0\}$;

(ii) the system $g_{i}(x, \omega) \leqslant 0, i=1, \ldots, m$, is regular;

(iii) the set $\operatorname{Sol}(\omega)$ is a singleton.

Proof. Necessity. To prove (i) we assume the contrary that there exists $\bar{v} \in \mathcal{H}, \bar{v} \neq 0$ such that

$$
T_{i} \bar{v}=0,\left\langle c_{i}, \bar{v}\right\rangle \leqslant 0, i=1, \ldots, m,\langle\bar{v}, T \bar{v}\rangle \leqslant 0
$$

Since $F(\omega) \neq \emptyset$, it follows from (22) that $F(\omega)$ is unbounded. For $\varepsilon>0$, put $T^{\varepsilon}=T-\varepsilon I$, where $I$ is the identity operator on $\mathcal{H}$. It follows from Lemma 2.6 that $\left\langle x, T^{\varepsilon} x\right\rangle$ is a Legendre form for $\varepsilon>0$ small enough. We can check that $\left\langle\bar{v}, T^{\varepsilon} \bar{v}\right\rangle<0$. Let $\omega^{k}=\left(T^{\varepsilon}, c, T_{1}, \ldots, T_{m}, c_{1}, \ldots, c_{m}, \alpha_{1}, \ldots, \alpha_{m}\right)$. Then, for any $x \in F\left(\omega^{k}\right)$,

$$
f\left(x+t \bar{v}, \omega^{k}\right)=\frac{1}{2}\left\langle x+t \bar{v}, T^{\varepsilon}(x+t \bar{v})\right\rangle+\langle c, x+t \bar{v}\rangle \rightarrow-\infty \text { as } t \rightarrow \infty .
$$

Thus $\operatorname{Sol}\left(\omega^{k}\right)=\emptyset$. This contradicts our assumption that $\operatorname{Sol}(\cdot)$ is lower semicontinuous at $\omega$. If (ii) does not hold, then one can find an $\omega^{k}$ arbitrarily close to $\omega$ such that

$$
F\left(\omega^{k}\right)=\left\{x \in \mathcal{H} \mid g_{i}\left(x, \omega^{k}\right) \leqslant 0\right\} \text { is empty }
$$

Hence, we can find a parameter $\omega^{k}=\left(T, c, T_{1}^{k}, \ldots, T_{m}^{k}, c_{1}^{k}, \ldots, c_{m}^{k}, \alpha_{1}^{k}, \ldots, \alpha_{m}^{k}\right)$ that close to $\omega=$ $\left(T, c, T_{1}, \ldots, T_{m}, c_{1}, \ldots, c_{m}, \alpha_{1}, \ldots, \alpha_{m}\right)$ such that $F\left(\omega^{k}\right)$ is empty. Since $\operatorname{Sol}\left(\omega^{k}\right)=\emptyset, \operatorname{Sol}(\cdot)$ cannot be lower semicontinuous at $\omega$. 
Suppose that $\operatorname{Sol}(\cdot)$ is lower semicontinuous at $\omega$, but $\operatorname{Sol}(\omega)$ is not a singleton. Since $\operatorname{Sol}(\omega) \neq \emptyset$, there exist $\bar{x}, \bar{y} \in \operatorname{Sol}(\omega)$ such that $\bar{x} \neq \bar{y}$. By the Hahn-Banach Theorem (see [4, Theorem 1.7]) there exists $\bar{c} \in \mathcal{H}$ such that

$$
\|\bar{c}\|=1, \quad\langle\bar{c}, \bar{x}\rangle>\langle\bar{c}, \bar{y}\rangle
$$

Clearly, there exists an open neighborhood $U$ of $\bar{x}$ such that

$$
\langle\bar{c}, x\rangle>\langle\bar{c}, \bar{y}\rangle \text { for all } \quad x \in U
$$

Given any $\delta>0$, we fix a number $\varepsilon \in(0, \delta)$ and put $c^{\varepsilon}=c+\varepsilon \bar{c}$. By (23) $),\left\|c^{\varepsilon}-c\right\|=\varepsilon<\delta$. Our next goal is to show that

$$
\operatorname{Sol}\left(\omega^{\varepsilon}\right)=\operatorname{Sol}\left(T, c^{\varepsilon}, T_{1}, \ldots, T_{m}, c_{1}, \ldots, c_{m}, \alpha_{1}, \ldots, \alpha_{m}\right) \cap U=\emptyset
$$

For any $x \in F(\omega) \cap U$, since $\bar{x}, \bar{y} \in \operatorname{Sol}(\omega)$, by (24) we have

$$
\begin{aligned}
\frac{1}{2}\langle x, T x\rangle+\left\langle c^{\varepsilon}, x\right\rangle & =\frac{1}{2}\langle x, T x\rangle+\langle(c+\varepsilon \bar{c}), x\rangle=\left(\frac{1}{2}\langle x, T x\rangle+\langle c, x\rangle\right)+\varepsilon\langle\bar{c}, x\rangle \\
& \geqslant \frac{1}{2}\langle\bar{x}, T \bar{x}\rangle+\langle c, \bar{x}\rangle+\varepsilon\langle\bar{c}, x\rangle>\frac{1}{2}\langle\bar{y}, T \bar{y}\rangle+\langle c, \bar{y}\rangle+\varepsilon\langle\bar{c}, \bar{y}\rangle \\
& =\langle\bar{y}, T \bar{y}\rangle+\left\langle c^{\varepsilon}, \bar{y}\right\rangle .
\end{aligned}
$$

It follows that $x \notin \operatorname{Sol}\left(\omega^{\varepsilon}\right)$. Thus, for the chosen neighborhood $U$ of $\bar{x} \in \operatorname{Sol}\left(\omega^{\varepsilon}\right)$ and for every $\delta>0$, there exists $c^{\varepsilon} \in \mathcal{H}$ satisfying $\left\|c^{\varepsilon}-c\right\|<\delta$ and $\operatorname{Sol}\left(\omega^{\varepsilon}\right) \cap U=\emptyset$. This contradicts the lower semicontinuity of $\operatorname{Sol}(\cdot)$. Hence $\operatorname{Sol}(\omega)$ is a singleton.

Sufficiency. Let $U$ be an open set in $\mathcal{H}$ containing the unique solution $\bar{x} \in \operatorname{Sol}(\omega)$. By (ii), there exists $\delta_{1}>0$ such that $F\left(\omega^{\prime}\right) \neq \emptyset$, for every $\omega^{\prime} \in \Omega$ satisfying $\left\|\omega^{\prime}-\omega\right\|<\delta_{1}$.

By (i), and Lemmas $2.6,2.5$, there exists $\delta_{2}$ such that for every

$$
\left(T^{\prime}, T_{1}^{\prime}, \ldots, T_{1}^{\prime}, c_{1}^{\prime}, \ldots, c_{m}^{\prime}\right) \in \mathcal{L}(\mathcal{H})^{m+1} \times \mathcal{H}^{m}
$$

satisfying

$$
\max \left\{\left\|T^{\prime}-T\right\|,\left\|T_{1}^{\prime}-T_{1}\right\|, \ldots,\left\|T_{m}^{\prime}-T_{m}\right\|,\left\|c_{1}^{\prime}-c_{1}\right\|, \ldots,\left\|c_{m}^{\prime}-c_{m}\right\|\right\}<\delta_{2},
$$

$\left\langle x, T^{\prime} x\right\rangle$ is a Legendre form and $\operatorname{Sol}\left(Q P R_{\omega^{\prime}}\right)=\{0\}$. Let $\delta:=\min \left\{\delta_{1}, \delta_{2}\right\}$. By Lemma 2.2, for every $\omega^{\prime}$ satisfying

$$
\left\|\omega^{\prime}-\omega\right\| \leqslant \delta
$$

we have $\operatorname{Sol}\left(\omega^{\prime}\right) \neq \emptyset$. From (i), (ii) and Theorem 2.1 it follows that $\operatorname{Sol}(\cdot)$ is upper semicontinuous at $\omega$. Hence, for $\delta>0$ small enough, $\operatorname{Sol}\left(\omega^{\prime}\right) \subset U$ for every $\omega^{\prime}$ satisfying (25)).

For such an $\delta>0$, from what has been said it follows that $\operatorname{Sol}\left(\omega^{\prime}\right) \cap \Omega \neq \emptyset$ for every $\omega^{\prime}$ satisfying (25). This shows that $\operatorname{Sol}(\cdot)$ is lower semicontinuous at $\omega$. The proof is complete. 
The following example shows that the assumption on the Legendre property of the quadratic form cannot be dropped from the assumption of Theorem 2.2 .

Example 2.4. Consider the problem (8) in Example 2.1, Let $\operatorname{Sol}(\omega)$ denote the solution set of (8) . It follows from Example 2.1 that $\operatorname{Sol}(\omega)$ is unbounded.

Let $\omega^{\varepsilon}=\left(T^{\varepsilon}, T_{1}, \alpha\right)$ where $T^{\varepsilon}=0+\varepsilon E, \varepsilon>0$ and $E$ is the identity operator on $L_{2}[0,1]$. Since $\left\|T^{\varepsilon}-T\right\|=\varepsilon$, we have

$$
\left\|\omega^{\varepsilon}-\omega\right\|=\max \left\{\left\|T^{\varepsilon}-T\right\|,\left\|T_{1}-T_{1}\right\|,\left|\alpha_{1}-\alpha_{1}\right|\right\}=\left\|T^{\varepsilon}-T\right\| \rightarrow 0 \text { as } \varepsilon \rightarrow 0 .
$$

Consider the problem

$$
\left\{\begin{array}{l}
\min f\left(x, \omega^{\varepsilon}\right)=\frac{\varepsilon}{2}\|x\|^{2} \\
\text { s. t. } x \in L_{2}[0,1], g_{1}\left(x, \omega^{\varepsilon}\right)=\frac{1}{2}\left\langle x, T_{1} x\right\rangle+\alpha_{1} \leqslant 0 .
\end{array}\right.
$$

Let $\operatorname{Sol}\left(\omega^{\varepsilon}\right)$ denote the solution set of $(26)$. It is clear that $\operatorname{Sol}\left(\omega^{\varepsilon}\right)=\{0\}$. Let $V$ be an open set in $\mathcal{H}$ such that $V \cap \operatorname{Sol}(\omega) \neq \emptyset$ and $0 \notin V$. Then, $V \cap \operatorname{Sol}\left(\omega^{\varepsilon}\right)=\emptyset$. Hence $\operatorname{Sol}(\cdot)$ is not lsc at $\omega=\left(T, T_{1}, \alpha_{1}\right)$.

Corollary 2.3. Consider the problem $Q P_{\omega}$ where $\langle x, T x\rangle$ is a nonpositive Legendre form, then the multifunction $\operatorname{Sol}($.$) is lower secontinuous at \omega$ if and only if the following conditions are satisfied:

(i) $F(\omega)$ is a compact set;

(ii) the system $g_{i}(x, \omega) \leqslant 0, i=1, \ldots, m$, is regular;

(iii) the set $\operatorname{Sol}(\omega)$ is a singleton.

Proof. Suppose that $\operatorname{Sol}(\cdot)$ is lower semicontinuous at $\omega$. Since $\operatorname{Sol}(\cdot)$ is lower semicontinuous at $\omega$, by Theorem 2.2 , conditions (ii) and (iii) are satisfied and $\left.\operatorname{Sol} Q P R_{\omega}\right)=\{0\}$. We now claim that $0^{+} F(\omega)=\{0\}$. Indeed, by $\langle x, T x\rangle$ is nonpositive, we have $\langle v, T v\rangle \leqslant 0$ for every $v \in 0^{+} F(\omega)$. If there exists no $\bar{v} \in 0^{+} F(\omega)$ with property that $\langle\bar{v}, T \bar{v}\rangle<0$ then $\left.\operatorname{Sol} Q P R_{\omega}\right)=\{0\}=0^{+} F(\omega)$. If $\langle\bar{v}, T \bar{v}\rangle<0$ for some $\bar{v} \in 0^{+} F(\omega)$ then it is obvious that $\operatorname{Sol} Q P R_{\omega}=\emptyset$, which is impossible. Our claim is proved. Since $\langle x, T x\rangle$ is a nonpositive Legendre form, $\mathcal{H}$ is of finite dimension (see, [9, Theorem 11.2]). Hence, since $F(\omega)$ is a nonempty closed set and $0^{+} F(\omega)=\{0\}, F(\omega)$ is a compact set.

Conversely, suppose that (i), (ii) and (iii) are satisfied. As $F(\omega) \neq \emptyset$ by assumption (i) implies that $0^{+} F(\omega)=\{0\}$. Therefore, $\left.\operatorname{Sol} Q Q P R_{\omega}\right)=\{0\}$. Since the conditions (i), (ii) and (iii) in Theorem 2.2 are satisfied, we conclude that $\operatorname{Sol}(\cdot)$ is lsc at $\omega$. The proof is complete

\section{Continuity of the Optimal Value Function}

We now present a set of two conditions which is necessary and sufficient for the continuity of $\varphi$ at a point $\omega$ where $\varphi$ has a finite value. 
Theorem 3.1. Consider the problem $\left(Q P_{\omega}\right)$ where $\langle x, T x\rangle$ is a Legendre form. Let $\omega \in \Omega$. Suppose that $\varphi(\omega) \neq \pm \infty$. Then, the optimal value function $\varphi(\cdot)$ is continuous at $\omega$ if and only if the following two conditions are satisfied:

(i) $\operatorname{Sol}\left(Q P R_{\omega}\right)=\{0\}$;

(ii) The system $g_{i}(x, \omega) \leqslant 0, i=1, \ldots, m$, is regular.

Proof. Suppose that $\varphi(\cdot)$ is continuous at $\omega$ and $\varphi(\omega) \neq \pm \infty$.

If the system $g_{i}(x, \omega) \leqslant 0, i=1, \ldots, m$, is irregular, then by Remark 2.1 there exists a sequence

$$
\omega^{k}:=\left\{\left(T_{1}^{k}, \ldots, T_{m}^{k}, c_{1}^{k}, \ldots, c_{m}^{k}, \alpha_{1}^{k}, \ldots, \alpha_{m}^{k}\right)\right\} \in \mathcal{L}(\mathcal{H})^{m} \times \mathcal{H}^{m} \times \mathbb{R}^{n}
$$

converging to $\omega:=\left(T_{1}, \ldots, T_{m}, c_{1}, \ldots, c_{m}, \alpha_{1}, \ldots, \alpha_{m}\right)$ such that, for every $k$,

$$
g_{i}\left(x, \omega^{k}\right):=\frac{1}{2}\left\langle x, T_{i}^{k} x\right\rangle+\left\langle c_{i}^{k}, x\right\rangle+\alpha_{i}^{k} \leqslant 0, i=1, \ldots, m
$$

has no solution. Since $F\left(\omega^{k}\right)$ is empty for every $k, \varphi\left(\omega^{k}\right)=+\infty$. On the other hand, since $\varphi(\cdot)$ is continuous at $\omega, \varphi(\omega)=\lim _{k \rightarrow \infty} \varphi\left(\omega^{k}\right)=+\infty$. We have arrived at a contradiction. This shows that $g_{i}(x, \omega) \leqslant 0$ is regular.

We now suppose that $\operatorname{Sol}\left(Q P R_{\omega}\right) \neq\{0\}$. Then, there is a nonzero $\bar{v} \in \mathcal{H}$ such that

$$
\langle\bar{v}, T \bar{v}\rangle \leqslant 0, \quad T_{i} \bar{v}=0,\left\langle c_{i}, \bar{v}\right\rangle \leqslant 0, \quad i=1, \ldots, m
$$

Define $T^{k}=T-\frac{1}{k} E$, where $E$ is the identity operator on $\mathcal{H}$. It follows from Lemma 2.6 that $\left\langle x, T^{k} x\right\rangle$ is a Legendre form for $k$ large enough. It is easy to check that $\left\langle\bar{v}, T^{k} \bar{v}\right\rangle<0$. Consider the sequence $\left\{\omega^{k}\right\}$,

$$
\omega^{k}=\left(T^{k}, c, T_{1}, \ldots, T_{m}, c_{1}, \ldots, c_{m}, \alpha_{1}, \ldots, \alpha_{m}\right) .
$$

From the assumption $\varphi(\omega) \neq \pm \infty$, it follows that $F(\omega) \neq \emptyset$. Hence, for any $x \in F(\omega)$ and for any $t>0$, we have $x+t \bar{v} \in F(\omega)$ and

$$
f\left(x+t \bar{v}, \omega^{k}\right)=\frac{1}{2}\left\langle x+t \bar{v}, T^{k}(x+t \bar{v})\right\rangle+\langle c, x+t \bar{v}\rangle \rightarrow-\infty \text { as } t \rightarrow \infty .
$$

This implies that, for every $k$ large enough, $\operatorname{Sol}\left(\omega^{k}\right)=\emptyset$ and $\varphi\left(\omega^{k}\right)=-\infty$. We arrived at a contradiction, because $\varphi(\cdot)$ is continuous at $\omega, \omega^{k}$ converges to $\omega$ and $\varphi(\omega) \neq \pm \infty$.

From now on we assume that (i) and (ii) are satisfied and $\omega^{k}$ is an arbitrarily sequence in $\mathcal{L}(\mathcal{H})^{m+1} \times$ $\mathcal{H}^{m+1} \times \mathbb{R}^{m}$ converging to $\omega$. By (ii) and Lemma 2.3, there exists a positive integer $k_{0}$ such that $F\left(\omega^{k}\right) \neq \emptyset$ for every $k \geqslant k_{0}$. By (i), Lemma 2.5] and Lemma 2.6, there exists positive integer $k_{1} \geqslant k_{0}$ such that for all $k \geqslant k_{1},\left\langle x, T^{k} x\right\rangle$ is a Legendre form and $\operatorname{Sol}\left(Q P R_{\omega^{k}}\right)=\{0\}$. It follows from Lemma 2.2 that $\operatorname{Sol}\left(\omega^{k}\right) \neq \emptyset$ with all $k$ large enough. Therefore, for every $k \geqslant k_{1}, \varphi\left(\omega^{k}\right)$ is finite. This means that, for every $k \geqslant k_{1}$, there exists $x^{k} \in \mathcal{H}$ satisfying

$$
\varphi\left(\omega^{k}\right)=\frac{1}{2}\left\langle x^{k}, T^{k} x^{k}\right\rangle+\left\langle c^{k}, x^{k}\right\rangle, g_{i}\left(x, \omega^{k}\right) \leqslant 0 .
$$


By (i) and Lemma 2.2, it follows that $\operatorname{Sol}(\omega) \neq \emptyset$. Taking any $x_{0} \in \operatorname{Sol}(\omega)$, we have

$$
\begin{aligned}
& \varphi(\omega)=\frac{1}{2}\left\langle x_{0}, T x_{0}\right\rangle+\left\langle c, x_{0}\right\rangle, \\
& g_{i}\left(x_{0}, \omega\right)=\frac{1}{2}\left\langle x_{0}, T_{i} x_{0}\right\rangle+\left\langle c_{i}, x_{0}\right\rangle+\alpha_{i} \leqslant 0, \quad i=1, \ldots, m .
\end{aligned}
$$

By (ii) and Lemma 2.3 , there exists a sequence $\left\{y_{k}\right\} \subset \mathcal{H}$ converging to $x_{0}$ and

$$
g_{i}\left(y_{k}, \omega\right)=\frac{1}{2}\left\langle y_{k}, T_{i} y_{k}\right\rangle+\left\langle c_{i}, y_{k}\right\rangle+\alpha_{i} \leqslant 0, i=1, \ldots, m \text { for every } k \geqslant k_{1} .
$$

From (27) it follows that $y_{k} \in F\left(\omega^{k}\right)$ for $k \geqslant k_{1}$. Then

$$
\varphi\left(\omega^{k}\right) \leqslant \frac{1}{2}\left\langle y_{k}, T^{k} y_{k}\right\rangle+\left\langle c^{k}, y_{k}\right\rangle
$$

It follows from (28) that

$$
\begin{aligned}
\limsup _{k \rightarrow \infty} \varphi\left(\omega^{k}\right) & \leqslant \limsup _{k \rightarrow \infty}\left(\frac{1}{2}\left\langle y_{k}, T^{k} y_{k}\right\rangle+\left\langle c^{k}, y_{k}\right\rangle\right)=\lim _{k \rightarrow \infty}\left(\frac{1}{2}\left\langle y_{k}, T^{k} y_{k}\right\rangle+\left\langle c^{k}, y_{k}\right\rangle\right) \\
& =\frac{1}{2}\left\langle x_{0}, T x_{0}\right\rangle+\left\langle c, x_{0}\right\rangle=\varphi(\omega) .
\end{aligned}
$$

We now claim that the sequence $\left\{x^{k}\right\}$ is bounded. Indeed, if it is unbounded then, by taking a subsequence if necessary, we can assume that $\left\|x^{k}\right\| \neq 0$ for every $k$ and $\left\|x^{k}\right\| \rightarrow \infty$ as $k \rightarrow \infty$. Then, the sequence $\left\{v^{k}\right\}=\left\{\left\|x^{k}\right\|^{-1} x^{k}\right\}$ is bounded. Without loss of generality, we can assume that $v^{k}$ itself weakly converges to some $v$. It is easy to check that $v \in 0^{+} F(\omega)$. By dividing both sides of the inequality

$$
\frac{1}{2}\left\langle x^{k}, T^{k} x^{k}\right\rangle+\left\langle c^{k}, x^{k}\right\rangle \leqslant \frac{1}{2}\left\langle y_{k}, T^{k} y_{k}\right\rangle+\left\langle c^{k}, y_{k}\right\rangle
$$

by $\left\|x^{k}\right\|^{2}$, letting $k \rightarrow \infty$ and by Lemma 2.4 , we get

$$
\langle v, T v\rangle \leqslant \liminf _{k \rightarrow \infty}\left\langle v^{k}, T^{k} v^{k}\right\rangle \leqslant \limsup _{k \rightarrow \infty}\left\langle v^{k}, T^{k} v^{k}\right\rangle \leqslant 0 .
$$

By an argument analogous to that used in the proof of Lemma 2.5, we have $v \in 0^{+} F(\omega) \backslash\{0\}$ and $\langle v, T v\rangle \leqslant 0$, contrary to (i). We have thus shown that the sequence $\left\{x^{k}\right\}$ is bounded; hence it has a weakly convergent subsequence. Without loss of generality, we may assume that $x^{k}$ weakly converges to $\bar{x}$. By Lemma 2.4, we get $\bar{x} \in F(\omega)$ and

$$
\liminf _{k \rightarrow \infty} \varphi\left(\omega^{k}\right)=\liminf _{k \rightarrow \infty}\left(\left\langle x^{k}, T^{k} x^{k}\right\rangle+\left\langle c^{k}, x^{k}\right\rangle\right) \geqslant\langle\bar{x}, T \bar{x}\rangle+\langle c, \bar{x}\rangle \geqslant \varphi(\omega) .
$$

Combining (29) with (30) gives $\lim _{k \rightarrow \infty} \varphi\left(\omega^{k}\right)=\varphi(\omega)$. This shows that $\varphi(\cdot)$ is continuous at $\omega$. The proof is complete.

Remark 3.1. Note that if the assumption on Legendre property of the quadratic form is omitted, then the problem $\left[Q P_{\omega}\right.$ may have no solution (see, [7, Example 3.3]). Hence the conclusion of Theorem 3.1 fails if the assumption on Legendre property of the quadratic form is omitted. 
Theorem 3.2. Consider the problem $\left(Q P_{\omega}\right)$ where $\langle x, T x\rangle$ is a Legendre form. The following four statements are equivalent:

$\left(\beta_{1}\right)$ the solution map $\operatorname{Sol}(\cdot)$ is lower semicontinuous at $\omega$;

$\left(\beta_{2}\right)$ the solution map $\operatorname{Sol}(\cdot)$ is continuous at $\omega$;

$\left(\beta_{3}\right) \operatorname{Sol}(\omega)$ is a singleton and the optimal value function $\varphi(\cdot)$ is Lipschitz continuous around $\omega$;

$\left(\beta_{4}\right) \operatorname{Sol}(\omega)$ is a singleton and the optimal value function $\varphi(\cdot)$ is continuous at $\omega$.

Proof. The equivalence between $\left(\beta_{1}\right)$ and $\left(\beta_{2}\right)$ follows immediately from the Theorem 2.1 and 2.2 . We next prove that $\left(\beta_{2}\right)$ implies $\left(\beta_{3}\right)$. Indeed, suppose that the solution map $\operatorname{Sol}(\cdot)$ is continuous at $\omega$. Then, solution map $\operatorname{Sol}(\cdot)$ is lower semicontinuous at $\omega$. It follows from Theorem 2.2 that $\operatorname{Sol}(\omega)$ is a singleton, $\left.\operatorname{Sol} Q P R_{\omega}\right)=\{0\}$ and the system $g_{i}(x, \omega) \leqslant 0$ is regular. It remains to show that $\varphi(\cdot)$ is locally Lipschitz at $\omega$. Since $f(\cdot, \cdot)$ is continuously differentiable at $(x, \omega)$, there exists $\delta>0$ so that $f$ is Lipschitz continuous with Lipschitz modulus $k_{f}>0$ on the set $\mathbb{U}_{\mathcal{H} \times \Omega}^{\delta}(x, \omega)$. By the regularity of the inequalities system $g_{i}(x, \omega) \leqslant 0$, the feasible set mapping $F(\cdot): \Omega \rightrightarrows \mathcal{H}$ is defined by $F\left(\omega^{\prime}\right)=\left\{x \in \mathcal{H} \mid g_{i}\left(x, \omega^{\prime}\right) \leqslant 0, i=1, \ldots, m\right\}$ has the Aubin property at $\omega$ for some $x \in F(\omega)$ (see, for instance, [5, Corollary 2.2]), that is, there exist $\varepsilon, \gamma, k_{F} \geqslant 0$ such that

$$
F\left(\omega^{1}\right) \cap U^{\gamma}(x) \subset F\left(\omega^{2}\right)+k_{F}\left\|\omega^{1}-\omega^{2}\right\| \mathbb{B}_{\Omega}(0,1)
$$

for every $\omega^{1}, \omega^{2} \in \mathbb{U}_{\Omega}^{\varepsilon}(\omega)$. Without loss of generality, we may assume that $\max \left\{\varepsilon, 2 \varepsilon k_{F}+\gamma\right\}<\delta$. Let $\omega^{1}, \omega^{2} \in \mathbb{U}_{\Omega}^{\varepsilon}(\omega)$ be chosen arbitrarily. By Lemma 2.3, the feasible set mapping $F(\cdot): \Omega \rightrightarrows \mathcal{H}$ is lower semicontinuous at $\omega$. This implies that $F\left(\omega^{1}\right) \neq \emptyset$ for $\varepsilon>0$ small enough. On the other hand, by $\operatorname{Sol} Q Q P R_{\omega}=\{0\}$, it follows that $\omega \in K$. Since $K$ is open, there exists $\varepsilon>0$ small enough such that $\omega^{1} \in K$. It follows from Lemma 2.2 that $\operatorname{Sol}\left(\omega^{1}\right) \neq \emptyset$, for $\varepsilon>0$ small enough. Hence there exists $x^{1} \in \operatorname{Sol}\left(\omega^{1}\right)$.

Since $\operatorname{Sol}(\cdot)$ is lsc at $\omega$, we can assume that $\varepsilon>0$ small enough to guarantee that $x^{1} \in U^{\gamma}(x)$. This implies that $x^{1} \in \operatorname{Sol}\left(\omega^{1}\right) \cap U^{\gamma}(x) \subset F\left(\omega^{1}\right) \cap U^{\gamma}(x)$.

Due to (31), there exists $x^{2} \in F\left(\omega^{2}\right)$ such that $\left\|x^{2}-x^{1}\right\| \leqslant k_{F}\left\|\omega^{2}-\omega^{1}\right\|$. From the choice of $\varepsilon$ and $\delta$ we derive:

$$
\begin{aligned}
\left\|\left(x^{1}, \omega^{1}\right)-(x, \omega)\right\| & =\max \left\{\left\|\omega^{1}-\omega\right\|,\left\|x^{1}-x\right\|\right\}<\max \{\varepsilon, \gamma\}<\delta, \\
\left\|\left(x^{2}, \omega^{2}\right)-(x, \omega)\right\| & =\max \left\{\left\|\omega^{2}-\omega\right\|,\left\|x^{2}-x\right\|\right\} \\
& \leqslant \max \left\{\left\|\omega^{2}-\omega\right\|,\left\|x^{2}-x^{1}\right\|+\left\|x^{1}-x\right\|\right\} \\
& \leqslant \max \left\{\left\|\omega^{2}-\omega\right\|, k_{F}\left\|\omega^{2}-\omega^{1}\right\|+\left\|x^{1}-x\right\|\right\} \\
& <\max \left\{\varepsilon, 2 \varepsilon k_{F}+\gamma\right\}<\delta .
\end{aligned}
$$

Thus $\left(x_{1}, \omega_{1}\right)$ and $\left(x_{2}, \omega_{2}\right)$ belong to $\mathbb{U}_{\mathcal{H} \times \Omega}^{\delta}(x, \omega)$.

Since $f$ is Lipschitz continuous on $\mathbb{U}_{\mathcal{H} \times \Omega}^{\delta}(x, \omega)$, we finally get

$$
\begin{aligned}
\varphi\left(\omega_{2}\right)-\varphi\left(\omega_{1}\right) & \leqslant f\left(\omega_{1}, x_{2}\right)-f\left(\omega_{1}, x_{1}\right) \leqslant k_{f} \max \left\{\left\|\omega_{2}-\omega_{1}\right\| ;\left\|x_{2}-x_{1}\right\|\right\} \\
& \leqslant k_{f} \max \left\{\left\|\omega_{2}-\omega_{1}\right\| ; k_{F}\left\|\omega_{2}-\omega_{1}\right\|\right\}=\max \left\{k_{f} ; k_{f} k_{F}\right\}\left\|\omega_{2}-\omega_{1}\right\| .
\end{aligned}
$$


Changing the roles of $x_{1}$ and $x_{2}$, we can obtain

$$
\varphi\left(\omega_{1}\right)-\varphi\left(\omega_{2}\right) \leqslant \max \left\{k_{f} ; k_{f} k_{F}\right\}\left\|\omega_{2}-\omega_{1}\right\|
$$

Hence $\varphi$ is Lipschitz continuous around $\omega$ with modulus $\max \left\{k_{f} ; k_{f} k_{F}\right\}$.

Clearly, $\left(\beta_{3}\right)$ implies $\left(\beta_{4}\right)$.

Finally, the implication $\left(\beta_{4}\right)$ implies $\left(\beta_{1}\right)$ follows from Theorem 2.2 and 3.1 . The proof is complete.

Example 3.1. We consider the problem $\left.Q P_{\omega}\right)$ with $\Omega=\mathcal{L}\left(\ell^{2}\right) \times \ell^{2} \times \mathcal{L}\left(\ell^{2}\right) \times \ell^{2} \times \mathbb{R}$.

Let $\omega=\left(T, c, T_{1}, c_{1}, \alpha_{1}\right)$, where $T: \ell^{2} \rightarrow \ell^{2}$ is defined by $T x=\left(0,-x_{2}, x_{3}, \ldots\right), c=(1,0, \ldots)$, $T_{1}: \ell^{2} \rightarrow \ell^{2}$ is defined by $T_{1} x=\left(x_{1}, x_{2}, \ldots\right), c_{1}=(0,0, \ldots), \alpha_{1}=-\frac{1}{2}$. This problem can be rewritten as follows

$$
\min _{x \in F(\omega)} f(x, \omega)=\frac{1}{2}\left(-x_{2}^{2}+x_{3}^{2}+\ldots x_{n}^{2}+\ldots\right)+x_{1}
$$

where $F(\omega)=\left\{\left(x_{1}, x_{2}, \ldots\right) \in \ell^{2} \mid g_{1}(x, \omega)=\frac{1}{2}\left(x_{1}^{2}+x_{2}^{2}+\ldots\right)-\frac{1}{2} \leqslant 0\right\}$.

Since $\langle x, T x\rangle=\left(x_{1}^{2}+x_{2}^{2}+x_{3}^{2}+\ldots+x_{n}^{2}+\ldots\right)-\left(x_{1}^{2}+2 x_{2}^{2}\right)$, by Proposition 3.79 in [3], $\langle x, T x\rangle$ is a Legendre form.

For $x=(0,0, \ldots)$, we have $g_{1}(0, \omega)=-\frac{1}{2}<0$. Hence $F(\omega) \neq 0$ and $g_{1}(x, \omega) \leqslant 0$ is regular.

We have $0^{+} F(\omega)=\left\{\left(v_{1}, \ldots\right) \in \ell^{2} \mid T_{1} v=\left(v_{1}, v_{2}, \ldots\right)=0\right\}=\{0\}$. Therefore Sol $\left.Q P R_{\omega}\right)=\{0\}$.

It is easy to check that $f(x, \omega) \geqslant \frac{1}{2}\left(x_{1}+1\right)^{2}+\left(\frac{1}{2} x_{2}^{2}+x_{3}^{2}+\ldots\right)-1 \geqslant-1$ for all $x \in F(\omega)$ and $\operatorname{Sol}(\omega)=\{-1,0,0, \ldots\}$.

Hence, by Theorem 3.2 , the solution map $\operatorname{Sol}(\cdot)$ is continuous at $\omega$ and the optimal value function $\varphi(\cdot)$ is Lipschitz continuous around $\omega$.

Corollary 3.1. Consider the problem $Q P_{\omega}$ where $\langle x, T x\rangle$ is elliptic. Suppose that the system $g_{i}(x, \omega) \leqslant 0, i=1, \ldots, m$, is regular. Then, the following four statements are equivalent:

$\left(\beta_{1}\right)$ the solution map $\operatorname{Sol}(\cdot)$ is lower semicontinuous at $\omega$;

$\left(\beta_{2}\right)$ the solution map $\operatorname{Sol}(\cdot)$ is continuous at $\omega$;

$\left(\beta_{3}\right)$ the optimal value function $\varphi(\cdot)$ is Lipschitz continuous around $\omega$;

$\left(\beta_{4}\right)$ the optimal value function $\varphi(\cdot)$ is continuous at $\omega$.

The proof of this corollary is simple, so it is omitted.

\section{Conclusions}

By using the Legendre property of quadratic form, we established continuity properties of the global solution map and the optimal value function for quadratic programming problems under finitely many convex quadratic constraints in Hilbert spaces.

In connection with Theorem 2.2, the following question seems to be interesting: Is there any verifiable sufficient condition for the set $\operatorname{Sol}(\omega)$ to be a singleton? 


\section{Acknowledgments}

This research is funded by Vietnam National Foundation for Science and Technology Development (NAFOSTED) under grant number 101.01-2014.39. The author would like to thank Prof. Nguyen Nang Tam for valuable remarks and suggestions. The author would like to express my sincere thanks to the anonymous referees and editors for insightful comments and useful suggestions.

\section{References}

[1] Bauschke, H. H., Combettes, P. L.: Convex Analysis and Monotone Operator Theory in Hilbert Spaces, Springer (2011)

[2] Berge, C.: Topological Spaces, Oliver and Boyd (1963)

[3] Bonnans, J. F., Shapiro, A.: Perturbation Analysis of Optimization Problems. Springer (2000)

[4] Brezis, H.: Functional Analysis, Sobolev Spaces and Partial Differential Equations, Springer (2011)

[5] Dempe, S., Mehlitz, P.: Lipschitz continuity of the optimal value function in parametric optimization, J Glob Optim 61, 363-377 (2015)

[6] Dinh, N., Goberna, M. A., Lpez, M. A.: On stability of the feasible set in optimization problems, SIAM J. Optim. 20(5), 2254-2280 (2010)

[7] Dong, V. V., Tam, N. N.: On the solution existence for convex quadratic programming problems in Hilbert spaces, Taiwanese J. Math. 20(6), 1417-1436 (2016)

[8] Dong, V. V., Tam, N. N.: On the solution existence of nonconvex quadratic programming problems in Hilbert spaces, submitted to Acta Math Vietnam

[9] Hestenes, M. R.: Applications of the theory of quadratic forms in Hilbert space to the calculus of variations, Pacific J. Math. 1, 525-581 (1951)

[10] Ioffe, A. D., Tikhomirov, V. M.: Theory of Extremal Problems, North-Holland, Amsterdam (1978)

[11] Kim, D. S., Tam, N. N., Yen, N. D.: Solution existence and stability of quadratic of quadratically constrained convex quadratic programs. Optim. Lett. 6, 363-373 (2012)

[12] Lee, G. M., Tam, N. N., Yen, N. D.: Quadratic Programming and Affine Variational Inequalities: A Qualitative Study, Series: Nonconvex Optimization and Its Applications, Springer (2005) 
[13] Schochetman, I. E., Smith, R. L., Tsui, S. K.: Solution existence for infinite quadratic programming, Technical Report 97-10 (1997)

[14] Semple, J.: Infinite positive-definite quadratic programming in a Hilbert space, J. Optim.Theory Appl. 88, 743-749 (1996)

[15] Sivakumar, K. C., Swarna, J. M.: Explicit solutions of infinite quadratic programs, J. Appl. Math. Comput. 12, $211-218$ (2003)

[16] Tam, N. N.: On Continuity of the solution map in quadratic programming, Acta Math Vietnam 24, 47-61 (1999)

[17] Tam, N. N.: Continuity of the optimal value function in indefinite quadratic programming, J Glob Optim 23, 43-61 (2002) 